\title{
Global modes in a confined impinging jet: application to heat transfer and control
}

Received: 22 May 2009 / Accepted: 3 December 2009 / Published online: 30 March 2010

(C) Springer-Verlag 2010

\begin{abstract}
We investigate the stability and control of a plane, laminar jet impinging on a flat plate in a channel, a geometry used to cool down a hot wall with a cold air jet in many industrial configurations. The global stability analysis indicates that, even for a strong confinement, the two-dimensional (2-D) steady flow is unstable to three-dimensional (3-D), steady perturbations. In the simplest limit case where dilatation effects are neglected, we show that the development of the instability induces a significant spanwise modulation of the heat flux at the impacted wall. To control the leading global mode, we propose adjoint-based 3-D harmonic and 2-D steady forcing in the bulk or at the wall. We show for instance that the unstable mode is controllable using a spanwise uniform blowing at the upper wall, in a specific domain corresponding to the footprint of the upper recirculating bubble. These techniques are applied to a novel open-loop control, in which we introduce into the flow a small airfoil, modelled by the lift force it exerts on the flow.
\end{abstract}

Keywords Impinging jet · Global instability $\cdot$ Wavemaker $\cdot$ Control $\cdot$ Lift

\section{Introduction}

Jet impingement is widely used in many industrial and engineering applications when an intense and rapid heat transfer is desired, for instance the tempering of metal sheets, the cooling of electronic components and turbine blades, or the drying of papers and textiles. The main advantage of this technique lies in the high localization of the cooling, as the use of high-speed jets allows to remove a large amount of heat on the impinging surface, around the stagnation region. It has been generally acknowledged that the use of such jets enhances the local transfer by more than an order of magnitude, compared to classical wall boundary layers [18].

A large body of works has been devoted to the study of impinging jet flows, including the effect of the jet geometry (circular, rectangular or plane cross-section), the nature of the impacted surface (plane, inclined or with obstacles) and the Reynolds number at the jet exit ([21] for a review). In many applications, the jet is operated in the laminar or transitional regime, due to the smallness of the scales involved. The jet is also often

Communicated by T. Colonius

P. Meliga (凶), J.-M. Chomaz

LadHyX-CNRS, Ecole Polytechnique, 91128 Palaiseau, France

E-mail: philippe.meliga@ladhyx.polytechnique.fr

J.-M. Chomaz

E-mail: jean-marc.chomaz@ladhyx.polytechnique.fr

Present address:

P. Meliga

LFMI, Ecole Polytechnique Fédérale de Lausanne, 1015 Lausanne, Switzerland

E-mail: philippe.meliga@epfl.ch 
confined between the target surface and an opposing surface in which the jet orifice is located, so as to increase the efficiency of the heat transfer. The presence of a confining top surface then results in a complex flow in which the behavior of the free jet is coupled to that of the channel flow developing between the two surfaces.

Only a limited amount of studies is available about highly confined, low Reynolds number jets. The steady and unsteady dynamics of a laminar two-dimensional (2-D) jet impinging on a flat plate have been investigated numerically $[4,13]$ and experimentally [22] for various Reynolds numbers and levels of confinement $2 \leq H / e \leq 9$, where $H$ is the distance from the jet exit to the wall and $e$ is the jet width. In particular, the direct numerical simulations (DNS) carried out by Lee et al. [13] on the most confined configuration $(H / e=2)$ show that the flow bifurcates from a 2-D steady to a 2-D unsteady state at $R e \sim 1050$, where $R e$ is the Reynolds number built from the channel height and the centerline velocity. However, these studies fail to consider the three-dimensional (3-D) aspects of the flow, which are crucial when spanwise homogeneous transfers are desired. For instance, for automotive purposes, steel sheets are coated by a zinc layer in order to resist to oxidization. Moving steel strips are dipped into a bath of molten zinc and then cooled down by impinging jets before being rolled up and sent to car manufacturers. Inhomogeneous protective zinc layers are therefore detrimental to the whole manufacturing process and should be alleviated.

In this study, we carry out the global stability analysis of a strongly confined jet of height ratio $H / e=2$, and show that the 2-D steady solution is unstable to 3-D steady disturbances. We characterize the effect of the instability on the heat flux at the impacted wall, as a way to illustrate the practical effect of global modes in industrial configurations. We also investigate adjoint-based control strategies for this global instability, using techniques introduced by Hill [12]. We discuss forcing methods that can be either 2-D steady or 3-D harmonic. The results are applied to a novel control strategy where a small airfoil is introduced into the flow. This control acts through the lift component of the force it exerts on the flow and differs from the classical control by means of a small cylinder, which acts through the drag component of the force.

\section{Problem formulation}

We investigate the stability of a confined impinging jet under nominally planar 2-D boundary conditions. A sketch of the computational domain and of the cartesian coordinate system $(x, y, z)$ used is shown in Fig. 1a, with $x, y$ and $z$, the horizontal, vertical and transverse directions. The jet of width $e$ first develops in a vertical channel of height $h_{\infty}$. At its exit, it is confined between the impacted wall and a horizontal plate positioned parallel to the wall, so that a horizontal channel of length $l_{\infty}$ and height $H$ is formed between the two surfaces. The boundary $\Gamma_{\text {lat }}$ representing the solid walls of the vertical channel are located at $x= \pm e / 2$. The boundaries $\Gamma_{\text {low }}$ and $\Gamma_{\text {up }}$ corresponding to the impacted wall and to the confinement plate are located at $y=0$ and $y=H$, respectively. Finally, the inlet $\Gamma_{\text {in }}$ and outlet $\Gamma_{\text {out }}$ are located, respectively, at $y=H+h_{\infty}$ and $x= \pm\left(e / 2+l_{\infty}\right)$.
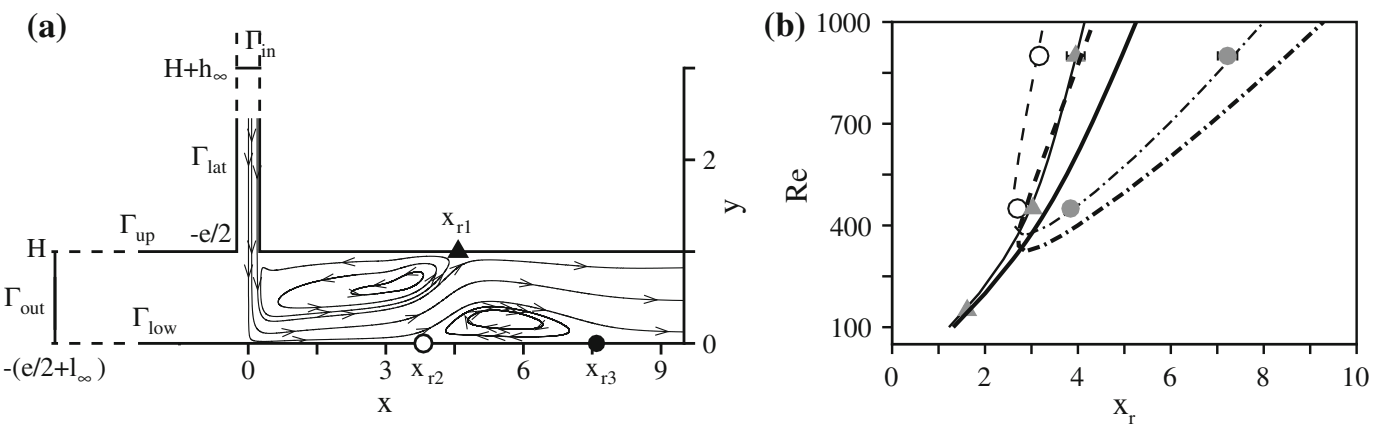

Fig. 1 a Schematic of the flow geometry. $h_{\infty}$ and $l_{\infty}$ denote the length of the vertical and horizontal channels, thus defining the location of the inlet $\Gamma_{\text {in }}$ and outlet $\Gamma_{\text {out }}$ boundaries at $y=H+h_{\infty}$ and $x= \pm\left(e / 2+l_{\infty}\right)$, respectively. The boundary $\Gamma_{\text {lat }}$ represents the solid walls of the vertical channel located at $x= \pm e / 2$. The boundaries $\Gamma_{\text {low }}$ and $\Gamma_{\text {up }}$ correspond to the impacted wall and to the confinement plate located, respectively, at $y=0$ and $y=H$. The rightmost part of the figure presents streamlines of the steady 2-D base flow at $R e=800$. The different separation and reattachment points $x_{r 1}, x_{r 2}$ and $x_{r 3}$ are indicated by the triangle and circle symbols. b Location of the separation and reattachment points $x_{r 1}$ (solid line), $x_{r 2}$ (dashed line) and $x_{r} 3$ (dash-dotted line) as a function of the Reynolds number. The thick black lines and the grey lines correspond, respectively, to the configuration studied in this article and to the truncated configuration studied by Chiriac and Ortega [4] and Lee et al. [13]. The symbols correspond to numerical values of the truncated configuration estimated from Fig. 6 of Lee et al. [13] 
In the following, we set the height ratio to $H / e=2$. All quantities are made non-dimensional using the height $H$ and the jet centerline velocity. The state vector $\boldsymbol{q}$ stands for the flow field $(\boldsymbol{u}, p)^{\mathrm{T}}$, where $\boldsymbol{u}=(u, v, w)^{\mathrm{T}}$ is the fluid velocity with $u, v$ and $w$, the $x, y$ and $z$-velocity components; $p$ is the pressure and ${ }^{\mathrm{T}}$ designates the transpose. The fluid motion is governed by the incompressible Navier-Stokes equations

$$
\nabla \cdot \boldsymbol{u}=0, \quad \partial_{t} \boldsymbol{u}+\nabla \boldsymbol{u} \cdot \boldsymbol{u}+\nabla p-R^{-1} \nabla^{2} \boldsymbol{u}=\mathbf{0} .
$$

We use a parabolic velocity profile $\boldsymbol{u}=(2 x-1)(2 x+1) \boldsymbol{e}_{y}$ at the inlet, no-slip conditions on the solid walls and a free outflow condition $-p \boldsymbol{n}+R e^{-1} \nabla \boldsymbol{u} \cdot \boldsymbol{n}$ at the outlet. Since the whole configuration is symmetric with respect to the jet axis of symmetry, all flow field quantities can be split into symmetric and antisymmetric fields. However, such symmetries are not explicitly imposed in this study, namely the whole configuration is meshed and no boundary conditions are imposed at the jet axis.

The state vector $\boldsymbol{q}$ is decomposed into a steady 2-D base flow $\boldsymbol{Q}$ and a 3-D perturbation $\boldsymbol{q}^{\prime}$ of infinitesimal amplitude $\epsilon$. The base flow is searched as a steady solution $\boldsymbol{Q}=(U, V, 0, P)^{\mathrm{T}}$ satisfying the 2-D equations

$$
\nabla \cdot \boldsymbol{U}=0, \quad \nabla \boldsymbol{U} \cdot \boldsymbol{U}+\nabla P-R^{-1} \nabla^{2} \boldsymbol{U}=\mathbf{0} .
$$

All perturbations are chosen in the form of normal eigenmodes of spanwise wavenumber $k$ and complex pulsation $\sigma+\mathrm{i} \omega, \sigma$ and $\omega$ being, respectively, the growth rate and the pulsation of the eigenmode ( $\sigma>0$ for an unstable eigenmode):

$$
\boldsymbol{q}^{\prime}=\hat{\boldsymbol{q}}(x, y) \mathrm{e}^{(\sigma+\mathrm{i} \omega) t+\mathrm{i} k z}+\text { c.c. }
$$

In (Eq. 3), $\hat{\boldsymbol{q}}=(\hat{u}, \hat{v}, \hat{w}, \hat{p})^{\mathrm{T}}$ is the so-called global mode, for which both the horizontal and the vertical directions $(x, y)$ are eigendirections, and c.c. denotes the complex conjugate of the preceding expression. This yields the generalized eigenvalue problem for $\lambda=\sigma+\mathrm{i} \omega$ and $\hat{\boldsymbol{q}}$ :

$$
\nabla \cdot \hat{\boldsymbol{u}}=0, \quad \lambda \hat{\boldsymbol{u}}+\nabla \hat{\boldsymbol{u}} \cdot \boldsymbol{U}+\nabla \boldsymbol{U} \cdot \hat{\boldsymbol{u}}+\nabla \hat{p}-R e^{-1} \nabla^{2} \hat{\boldsymbol{u}}=\mathbf{0}
$$

the boundary conditions consisting of a Dirichlet condition $\hat{\boldsymbol{u}}=\mathbf{0}$ at the inlet and the solid walls, and of a free outflow condition $-\hat{\boldsymbol{p}} \boldsymbol{n}+R e^{-1} \nabla \hat{\boldsymbol{u}} \cdot \boldsymbol{n}=\mathbf{0}$ at the outlet.

The FreeFem++ software ${ }^{1}$ is used to generate a mesh composed of triangular elements with the DelaunayVoronoi algorithm. The mesh refinement is controlled by the vertex densities on both external and internal boundaries. The base flow and disturbance equations are numerically solved by a finite-element method, using the same mesh. The unknown velocity and pressure fields are spatially discretized using a basis of Taylor-Hood elements, i.e. P2 elements ( 6 degrees of freedom) for velocities and P1 elements ( 3 degrees of freedom) for pressure. The associated variational formulations are derived and spatially discretized on the mesh, and the sparse matrices resulting from the projection of the variational formulations onto the basis of finite elements are built with the FreeFem++ software.

The base flow $\boldsymbol{Q}$, solution of the steady, 2-D, non-linear equations (2) is obtained using an iterative Newton method [1]. Starting from any guess value $\overline{\boldsymbol{Q}}$, presently obtained by time-marching a DNS of equations (1) along with the required boundary conditions, this method involves the resolution of simple linear problems. At each step, a matrix inversion is performed using the UMFPACK library, which consists in a sparse direct LU solver [7]. The spatial discretization of problem (4) results in a large-scale generalized eigenvalue problem, solved using the "Implicitly Restarted Arnoldi method" of the ARPACK library ${ }^{2}$ based upon a shift and invert strategy [8]. All global modes are normalized by imposing the phase of the $x$-velocity component to be zero at $x=10$ and $y=0.5$, i.e. $\hat{u}(10,0.5)$ is real positive. The eigenmode energy is then normalized to unity in a fixed domain $\Omega_{\text {in }}$ defined arbitrarily as $|x| \leq 8.125$ and $y \leq 3$, so that $\int_{\Omega_{\text {in }}}|\hat{\boldsymbol{u}}|^{2}(x, y) \mathrm{d} \Omega=1$. This normalization choice has no effect on the results presented here. It is simply used to test the convergence of complex eigenvectors when computed on various domain sizes.

\footnotetext{
1 http://www.freefem.org.

2 http://www.caam.rice.edu/software/ARPACK.
} 


\section{Results}

\subsection{Base flow computations}

The rightmost part of Fig. 1a shows the streamlines of the symmetric base flow computed at $R e=800$. It exhibits a first separation bubble extending at the upper wall from the edge of the nozzle to the horizontal position $x_{r 1}$, and a second separation bubble at the lower wall extending from $x_{r 2}$ to $x_{r 3}$. The location of these points, illustrated by the symbols sketched in Fig. 1a, is presented for various Reynolds numbers as the thick black solid, dashed and dash-dotted lines in Fig. 1b. We find that the second separation zone appears for $R e_{r 2} \simeq 330$. Close to this threshold value, the size of this second region, defined as $L_{r 2}=x_{r 3}-x_{r 2}$, scales as $L_{r 2} \propto\left(R e-R e_{r 2}\right)^{1 / 2}$ (not shown here). The grey lines in Fig. 1b correspond to base flow calculations carried out on the truncated configuration studied by Chiriac and Ortega [4] and Lee et al. [13], values estimated from this last study being also reported as the grey triangle and circle symbols. In their studies, these authors have set the height of the vertical channel to $h_{\infty}=0$ and have imposed a uniform velocity profile directly at the jet exit, i.e. flush with the upper wall, meaning that the dynamics of the free jet is no more coupled to that of the fluid in the horizontal channel. Our results precisely show that the location of the separation and reattachment points is very sensitive to the jet velocity profiles, as their position is systematically underestimated in the truncated configuration. This outlines the importance of including the vertical channel in the computation of impinging jet flows.

\subsection{Two-dimensional global modes}

We find that the base flow is stable to any 2-D disturbances in the range of Reynolds numbers under consideration: Fig. 2a shows the evolution of the growth rate of the two leading 2-D modes as a function of the Reynolds number, up to $R e=1400$. These modes are stationary $(\omega=0)$, and exhibit small negative growth rates $(\sigma<0$ and $|\sigma| \ll 1)$, meaning that they are very slowly damped. The leading global mode is antisymmetric, whereas the second leading mode is symmetric. We recall here that the symmetry of the modes has not been enforced in the numerics, but emerges spontaneously from the computations even with a non-symmetric computational grid. In the following, the eigenvectors corresponding to these antisymmetric and symmetric modes are referred to $\hat{\boldsymbol{q}}_{0, a}$ and $\hat{\boldsymbol{q}}_{0, s}$, respectively. The effect of these modes can be physically interpreted by considering the flow reconstructed as the linear superposition of the base flow $\boldsymbol{Q}$ and the global modes $\hat{\boldsymbol{q}}_{0, a}$ and $\hat{\boldsymbol{q}}_{0, s}$ with a finite amplitude $\epsilon$. For both the modes, $\epsilon$ has been set so that the energy of the global mode represents $0.25 \%$ that of the base flow. Figure 3 shows the separation lines of the base flow and of the total flow $\boldsymbol{Q}+\epsilon \hat{\boldsymbol{q}}$ as the dashed and solid lines, respectively, whereas the levels of energy of the total flow are indicated by the colour shading. The main effect of the 2-D modes is to shift the location of the separation and reattachment points (up to 9\% for the chosen perturbation amplitude). The slow dynamics associated to these weakly damped modes has therefore an important impact if one aims at obtaining precise approximations of the steady state using a time-marching procedure [4,13], since the simulation must last long enough for these
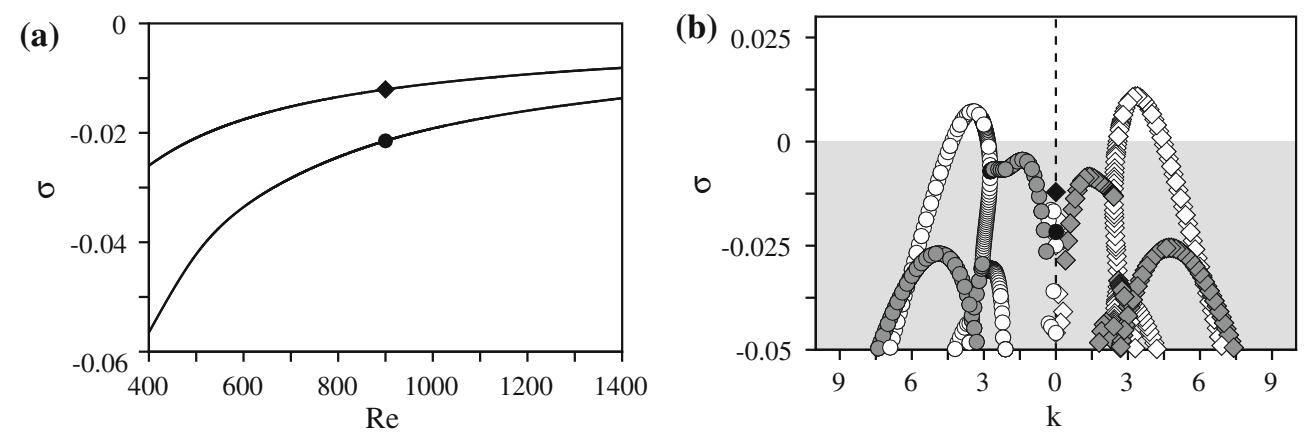

Fig. 2 a Growth rate of the two leading 2-D global modes as a function of the Reynolds number. The black symbols correspond to the Reynolds number $R e=900$ for which the complete eigenvalue spectrum is shown in (b). b Eigenvalue spectrum in the $(k, \sigma)$-plane for $R e=900$. The light grey shaded area stands for the stable domain $\sigma \leq 0$. The right and left half-planes correspond to antisymmetric and symmetric modes, respectively, whereas the white and dark grey symbols denote steady and oscillating modes. The black symbols correspond to the leading 2-D modes reported in (a) for this Reynolds number 
(a)

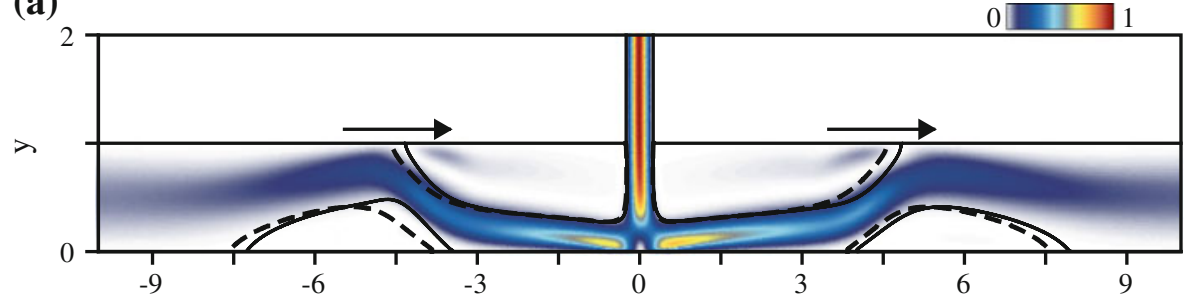

(b)

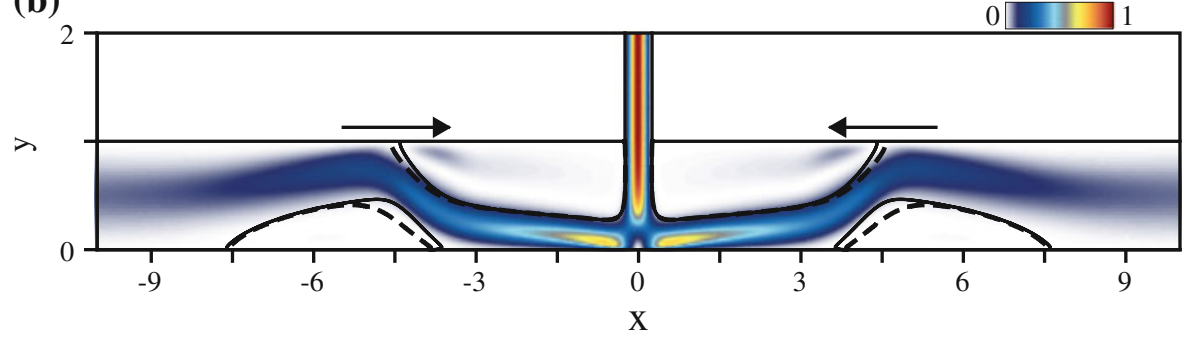

Fig. 3 Physical effect of the 2-D modes at $R e=800$. The dashed and solid lines stand for the separation lines of the base flow and of the total flow $\boldsymbol{Q}+\epsilon \hat{\boldsymbol{q}}$, respectively. $\epsilon$ has been set so that the perturbation energy represents $0.25 \%$ of the base flow energy. The colour shading depicts the spatial distribution of energy of the total flow, and the white hue corresponds to vanishing amplitudes. a Antisymmetric mode $\hat{\boldsymbol{q}}_{0, a}$, b symmetric mode $\hat{\boldsymbol{q}}_{0, s}$

modes to reach a small enough amplitude. For the amplitude to decay by $n$ orders of magnitude, the simulation should last at least $2.3 n /\left|\sigma_{0, \max }\right|$, where $\sigma_{0, \max }$ is the largest growth rates over all 2-D modes. Practically, for a Reynolds number $R e=800$ and $n=3$, i.e. a decrease of $10^{-3}$ in the amplitude of the departure from the exact steady state, this requires to run the numerical simulation for more than 500 times the advection time scale. This justifies the present use of the Newton iterative algorithm, which allows to compute steady states in about five iterations only, the residual of the governing equations being then smaller than $10^{-12}$.

\subsection{3-D global modes}

Figure $2 \mathrm{~b}$ presents the eigenvalue spectrum for both 2-D and 3-D modes at the Reynolds number $R e=900$, the light grey shaded area standing for the stable domain $\sigma \leq 0$. Antisymmetric and symmetric modes are plotted as diamonds and circles in the right and left half-planes, respectively, whereas white and dark grey symbols denote stationary and oscillating global modes. The eigenvalues associated to the 2-D modes $\hat{\boldsymbol{q}}_{0, a}$ and $\hat{\boldsymbol{q}}_{0, s}$ are also reported as the black symbols. In practice, such a distinction between antisymmetric and symmetric modes has been achieved by computing first this spectrum on the full configuration illustrated in Fig. 1a. It has been then recomputed on half the configuration along with symmetric and antisymmetric boundary conditions at the jet axis, both spectra being identical. The growth rate $\sigma$ is positive for a branch of stationary, antisymmetric modes of spanwise wavenumbers in the range $2.57 \leq k \leq 4.46$ (respectively for a branch of stationary, symmetric modes of spanwise wavenumbers in the range $2.83 \leq k \leq 4.33$ ), meaning that the 2-D steady flow is unstable to 3-D disturbances, even though the Reynolds number is lower than the threshold value reported by [13] for the transition to the 2-D unsteady state. The threshold parameters relative to both branches have been computed: we find that the antisymmetric branch becomes unstable for the Reynolds number $R e_{a}=858$ and a spanwise wavenumber $k_{a}=3.49$ corresponding to a wavelength $2 \pi / k_{a}=1.80$, (respectively the symmetric branch becomes unstable for $R e_{s}=871.5$ and $k_{s}=3.57$, i.e. $2 \pi / k_{s}=1.76$ ). In the following, the eigenvectors corresponding to these antisymmetric and symmetric modes are referred to as $\hat{\boldsymbol{q}}_{k, a}$ and $\hat{\boldsymbol{q}}_{k, s}$, respectively. Figure 4 shows the spatial structure of the $x$-velocity component $\hat{u}_{k, a}$ of the antisymmetric mode, which is dominated by axially extended disturbances located downstream of the stagnation point. The $x$-velocity component $\hat{u}_{k, s}$ of the symmetric mode turns out to be amazingly similar (not shown here for conciseness). This similarity in the structure, as well as the comparable growth rates $\sigma_{k, a}$ and $\sigma_{k, s}$ of both modes, suggests that both the right and the left half-flows are able to sustain a 3-D global instability, the disturbances growing in each half-domain with little interaction with the other half-domain. Consequently, we restrict from now on the discussion to the 3-D antisymmetric mode so as to ease the reading, the results for the symmetric mode being similar. 


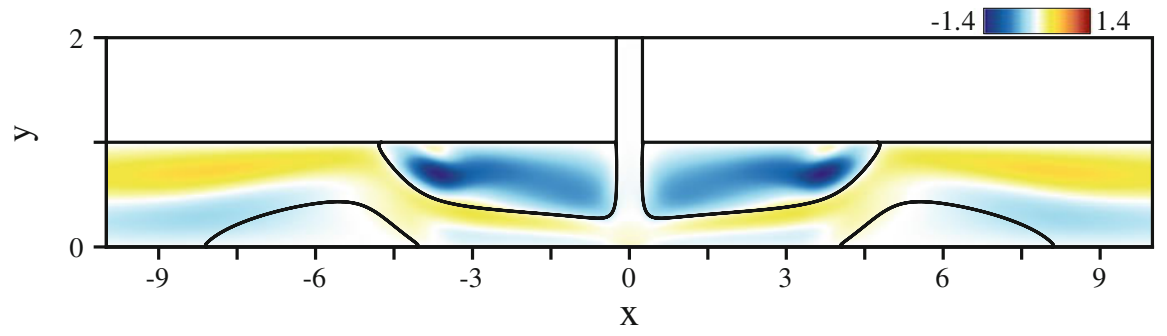

Fig. 4 Spatial distribution of the $x$-velocity component for the leading antisymmetric 3-D global mode $\hat{\boldsymbol{q}}_{k, a}$ at threshold of instability- $R e_{a}=858, k_{a}=3.49$

Identifying the physical mechanism underlying this 3-D instability remains challenging. In the context of local stability, different regions of interest can be identified that potentially lead to hyperbolic instability near the location of impingement (where the vorticity perturbation may be stretched in the hozizontal direction), centrifugal instability along the first recirculating bubble, and elliptic instability in the region between the first and the second recirculating bubbles (where the base flow presents a permanent elliptic vortex). However, since there is no scale separation between the variations of the unstable mode and that of the base flow, identifying such a local instability mechanism has not been possible. For instance, part of the streamlines of the first recirculating bubble corresponds to a negative Rayleigh discriminant [2] and is therefore unstable to centrifugal instability. However, the sign of the discriminant changes along the streamlines, so that the sufficient criterion for centrifugal instability fails $[9,20]$. However, the instability may result from cooperative effects involving several flow regions sensitive to different local instability mechanisms, but the WKBJ analysis [14] requiring to determine the evolution of a local wave vector along streamlines is out of the scope of this study. On the one hand, the local theory will provide only a qualitative description of the present global instability which occurs not at large but at moderate wavenumbers. On the other hand, the present global stability analysis is far more quantitative and will allow a precise identification of the flow regions responsible for the loss of stability, as will be discussed in the following.

\subsection{Effect on the wall heat transfer}

We are now interested in the effect of the 3-D global mode $\hat{\boldsymbol{q}}_{k, a}$ in terms of the physical quantity carried by the flow. Neglecting buoyancy effects, we choose here to study temperature viewed as a passive scalar (i.e. temperature evolves in a similar way than the mass fraction of a reactant in a chemical reaction), and investigate the wall heat transfer which is the physically meaningful quantity. We model a cooling set-up by considering a cold fluid of temperature $T_{\text {in }}$ at the inlet, and a hot lower wall of temperature $T_{\mathrm{w}}>T_{\text {in }}$, all other walls being perfectly isolating. All temperature quantities are made non-dimensional using the temperature difference $\delta T=T_{\mathrm{w}}-T_{\mathrm{in}}$. In the presence of an unstable mode, the temperature is split into a base component $\Theta$, modulated by a small perturbation $\hat{\theta}$ of amplitude $\epsilon$. The base component $\Theta$ is a nearly constant field that can be computed from the base flow velocity by solving the advection-diffusion problem

$$
\boldsymbol{U} \cdot \nabla \Theta-\frac{\gamma}{\operatorname{PrRe}} \nabla^{2} \Theta=\mathbf{0},
$$

where $\gamma$ is the ideal gas constant and $\operatorname{Pr}=\mu c_{p} / \kappa$ is the Prandtl number defined from the dynamic viscosity $\mu$, the specific heat $c_{p}$ and the thermal conductivity $\kappa$, set here to $\operatorname{Pr}=0.71$. Relation (5) is solved with Dirichlet conditions $\Theta=0$ at the inlet and $\Theta=1$ at the lower wall and a Neumann condition $\partial_{n} \Theta=0$ at all other boundaries. The perturbation field $\hat{\theta}$ that can be similarly computed from the global mode eigenvalue and velocity by solving the linearized problem

$$
\lambda \hat{\theta}+\boldsymbol{U} \cdot \nabla \hat{\theta}+\hat{\boldsymbol{u}} \cdot \nabla \Theta-\frac{\gamma}{\operatorname{PrRe}} \nabla^{2} \hat{\theta}=\mathbf{0},
$$

along with homogeneous boundary conditions.

The temperature fields associated to the marginally stable antisymmetric 3-D global mode are shown in Fig. 5. The base and disturbance components $\Theta$ and $\hat{\theta}_{k, a}$ are presented in Fig. 5a and b, respectively, the localization of the first and second recirculating bubbles being evidenced by the solid lines. Only the $x \geq 0$ 

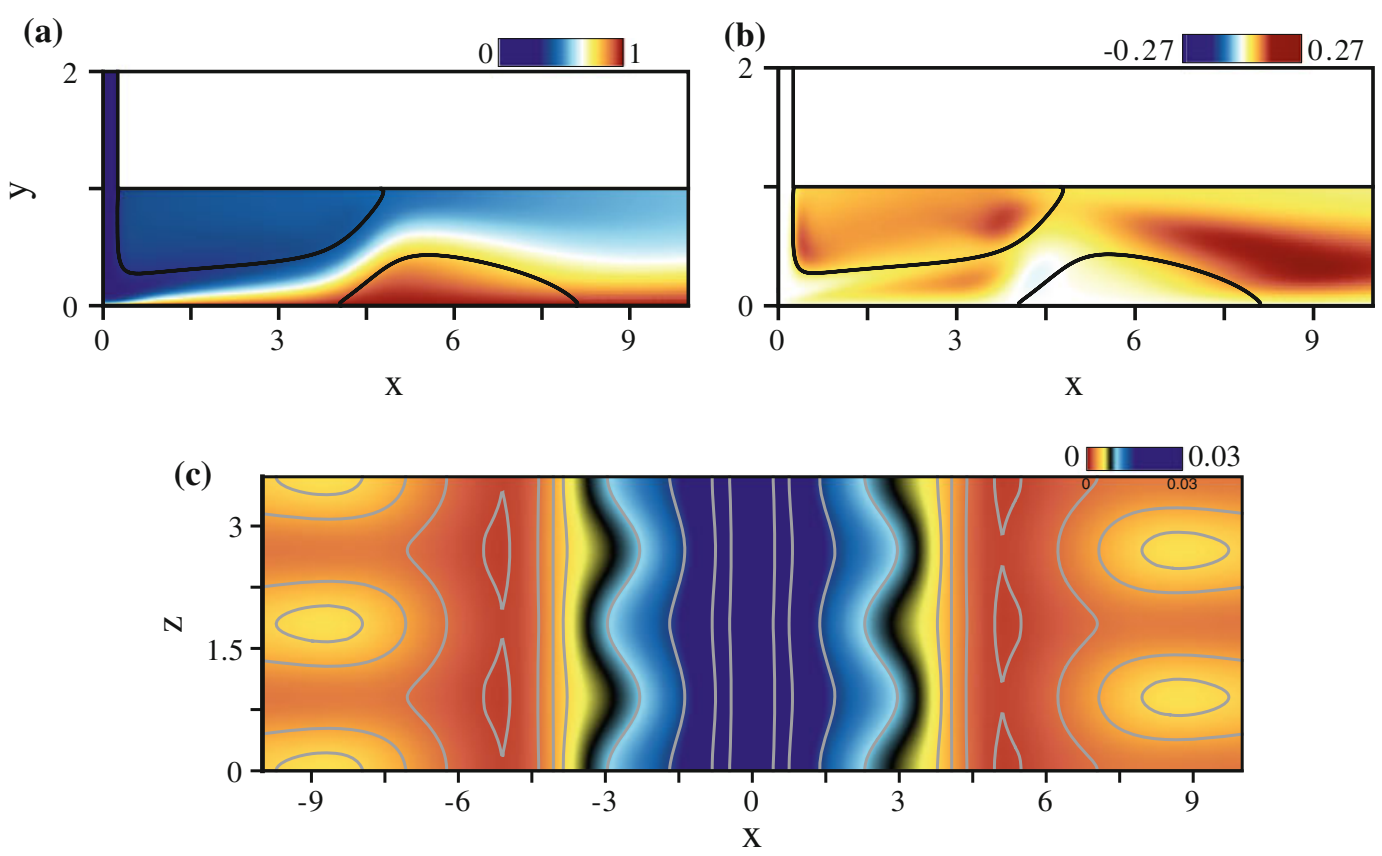

Fig. 5 Effect of the 3-D antisymmetric global mode $\hat{\boldsymbol{q}}_{k, a}$ on the wall heat transfer for a cooling set-up modelled by equations (5) and (6) $-R e_{a}=858, k_{a}=3.49$. a Base flow component of the temperature field $\Theta$, $\mathbf{b}$ disturbance component of the temperature field $\hat{\theta}_{k, a}$. The localization of the first and second recirculating bubbles is evidenced by the solid lines, $\mathbf{c}$ visualization of the total head flux $J+\epsilon \hat{j}_{k, a}$ at the impacted wall for a perturbation whose energy represents $1 \%$ of the base flow energy. The contours are enhanced by the grey solid lines

half-domain is presented, as we find that $\Theta$ and $\hat{\theta}_{k, a}$ are, respectively, symmetric and antisymmetric with respect to the jet axis. One observes strong temperature gradients for $\Theta$ in the sheared region close to the hot lower wall. The disturbance field $\hat{\theta}_{k, a}$ is most intense in the second recirculating bubble where hot gas has accumulated and extends far downstream, up to a distance of $20 \mathrm{H}$. The modulation of the wall heat flux resulting from the 3-D instability is illustrated in Fig. 5c, where we show a 2-D plot of the total heat flux $J+\epsilon \hat{j}_{k, a}$ evaluated at the impacted wall, with

$$
J=-\frac{\gamma}{\operatorname{PrRe}} \partial_{y} \Theta, \quad \text { and } \quad \hat{j}_{k, a}=-\frac{\gamma}{\operatorname{PrRe}} \partial_{y} \hat{\theta}_{k, a},
$$

$\epsilon$ being set so that the perturbation energy represents $1 \%$ of the base flow energy. The contours, enhanced by the grey solid lines, exhibit a significant spanwise inhomogeneity over a large distance of $10 \mathrm{H}$. Controlling this 3-D instability is therefore compulsory for most engineering applications, where one seeks the most uniform cooling to avoid non-homogeneous thermo-mechanical stresses or non-homogeneous temperature-dependent chemical reactions.

\section{Adjoint global modes and 3-D harmonic control}

In the perspective of control, we investigate now how the 3-D global mode $\hat{\boldsymbol{q}}_{k, a}$, taken at the threshold of instability, may be affected by imposing a forcing term of small amplitude, under the form of either a bulk force or a blowing and suction applied at a control surface $\Gamma_{\mathrm{c}}$ (which can be only the lateral and upper walls for the practical application, i.e. $\Gamma_{\mathrm{c}}=\Gamma_{\text {lat }} \cup \Gamma_{\text {up }}$ ).

We consider first a small-amplitude near-resonance harmonic forcing, occurring through a bulk force $\delta \hat{\boldsymbol{f}}$ or a blowing and suction velocity $\delta \hat{\boldsymbol{u}}_{W}$ of same frequency and spanwise wavenumber as the global mode, namely $\omega=0$ and $k=k_{a}$ here. We assume that the forcing acts at the perturbation level, so that the base flow equations (2) are unchanged. The disturbance equations now read

$$
\nabla \cdot \hat{\boldsymbol{u}}=0, \quad \lambda \hat{\boldsymbol{u}}+\nabla \hat{\boldsymbol{u}} \cdot \boldsymbol{U}+\nabla \boldsymbol{U} \cdot \hat{\boldsymbol{u}}+\nabla \hat{p}-R^{-1} \nabla^{2} \hat{\boldsymbol{u}}=\delta \hat{\boldsymbol{f}},
$$


along with the boundary condition $\hat{\boldsymbol{u}}=\delta \hat{\boldsymbol{u}}_{W}$ on $\Gamma_{\mathrm{c}}$. Under these assumptions, Giannetti and Luchini [11] have established that the receptivity of a damped global mode, i.e. its amplitude owing to forcing, is given by

$$
\mathcal{A}_{f}=\frac{1}{\sigma A} \int_{\Omega} \hat{\boldsymbol{u}}^{\dagger} \cdot \boldsymbol{\delta} \hat{\boldsymbol{f}} \mathrm{d} \Omega+\frac{1}{\sigma A} \int_{\Gamma_{\mathrm{c}}}\left(\hat{p}^{\dagger} \boldsymbol{n}+R e^{-1} \nabla \hat{\boldsymbol{u}}^{\dagger} \cdot \boldsymbol{n}\right) \cdot \delta \hat{\boldsymbol{u}}_{W} \mathrm{~d} l,
$$

with $\mathrm{d} l$ the length element along $\Gamma_{c}, A=\int_{\Omega} \hat{\boldsymbol{u}}^{\dagger} \cdot \hat{\boldsymbol{u}} \mathrm{d} \Omega$ and $\hat{\boldsymbol{q}}^{\dagger}=\left(\hat{\boldsymbol{u}}^{\dagger}, \hat{p}^{\dagger}\right)^{\mathrm{T}}$ the adjoint global mode, solution of the adjoint eigenvalue problem

$$
\nabla \cdot \hat{\boldsymbol{u}}^{\dagger}=0, \quad \lambda^{*} \hat{\boldsymbol{u}}^{\dagger}+\nabla \boldsymbol{U}^{\mathrm{T}} \cdot \hat{\boldsymbol{u}}^{\dagger}-\nabla \hat{\boldsymbol{u}}^{\dagger} \cdot \boldsymbol{U}+\nabla \hat{p}^{\dagger}-R e^{-1} \nabla^{2} \hat{\boldsymbol{u}}^{\dagger}=\mathbf{0}
$$

where the superscripted asterisk stands for the complex conjugate. From a physical point of view, investigating the adjoint quantities therefore allows to determine the flow region where forcing is able to produce the greatest global mode amplitude.

Equations (10) are obtained by integrating by part problem (4) and the boundary conditions to be fulfilled by adjoint perturbations are such that all boundary terms arising during the integration vanish [19]. We obtain a Dirichlet condition $\hat{\boldsymbol{u}}^{\dagger}=\mathbf{0}$ at the inlet and on the solid walls, and an adjoint outflow condition $(\boldsymbol{U} \cdot \boldsymbol{n}) \hat{\boldsymbol{u}}^{\dagger}+\hat{p}^{\dagger} \boldsymbol{n}+\operatorname{Re}^{-1} \nabla \hat{\boldsymbol{u}}^{\dagger} \boldsymbol{n}=\mathbf{0}$ at the outlet. The adjoint eigenproblem (10) is solved via the Arnoldi method already introduced for the computation of global modes, now also referred to as the direct global modes. Adjoint global modes are then normalized so that $A=\int_{\Omega} \hat{\boldsymbol{u}}^{\dagger} \cdot \hat{\boldsymbol{u}} \mathrm{d} \Omega=1$. We check a posteriori that the adjoint eigenvalues are complex conjugate with the direct eigenvalues and that the bi-orthogonality relation [5] is satisfied for the 10 leading global modes (i.e. that the scalar product of one of the 10 leading adjoint modes with any of the 10 leading direct modes is less that $10^{-7}$, except when the direct and adjoint modes are associated to complex conjugate eigenvalues). We conclude that our numerical procedure accurately estimates the direct and adjoint global modes; although, the discrete numerical procedures used to solve the direct and adjoint eigenproblems lead to discrete operators that are not exactly hermitian one to the other. It turns out that the direct global mode and its associated adjoint mode have the same symmetry, in particular the leading adjoint global mode $\hat{\boldsymbol{q}}_{k, a}^{\dagger}$ is also antisymmetric.

\subsection{Wall forcing}

In the case of wall forcing, relation (9) shows that the amplitude of the global mode is determined by two distinct contributions associated to mass and viscous effects. The product of the wall-normal component of the velocity $\delta \hat{\boldsymbol{u}}_{W} \cdot \boldsymbol{n}$ with the adjoint pressure $\hat{p}^{\dagger}$, taken at the wall, accounts for the effect of the mass flux, whereas the contribution weighted by the inverse of the Reynolds number takes into account the modification of the viscous force at the wall when blowing and suction is applied. Note that this latter term is the only one sensitive to velocities tangential to the wall. It has been argued in previous studies [16] and indeed checked in the present case that for sufficiently large Reynolds numbers, the effect of the viscous stress can be neglected compared to that of the mass flux, implying that the flow is receptive only to the wall-normal component of the velocity, and that the forcing is efficient only in regions of the wall where the magnitude of $\hat{p}^{\dagger}$ is large.

The distributions of adjoint pressure for the antisymmetric global mode $p_{k, a}^{\dagger}$, taken at the lateral and upper walls, are shown in Fig. 6a, where the dashed grey lines stand for the position of the nozzle edge. On the lateral wall (left half of Fig. 6a), the adjoint pressure distribution increases significantly close to the edge, where the maximum value is reached. On upper wall (right half of Fig. 6a), the magnitude of adjoint pressure remains almost zero everywhere except upstream of the first reattachment point, whose position is indicated by the solid grey line. However, the maximum value remains lower than that found on the lateral wall. Consequently, maximum efficiency will be roughly achieved by placing an actuator at the nozzle edge, that imposes a stationary $k=k_{a}$ normal wall velocity.

\subsection{Bulk forcing}

In the case of bulk forcing, the amplitude of the forced global mode is determined by the adjoint velocity field $\hat{\boldsymbol{u}}_{\boldsymbol{k}, \boldsymbol{a}}^{\dagger}$. Its $x$-component, presented in Fig. 6b, exhibits high magnitudes within the first recirculating bubble and small but finite values far upstream from the stagnation point, i.e. in the inlet section. In Fig. 6b, this 
(a)

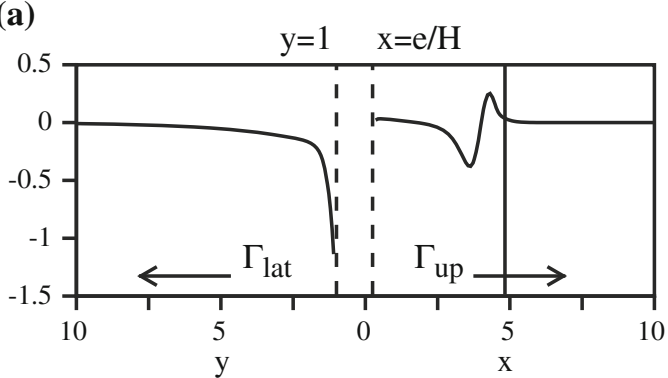

(b)

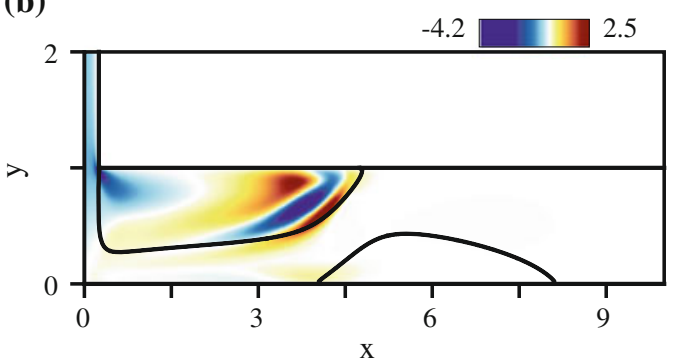

Fig. 6 Antisymmetric mode at the threshold of instability- $\operatorname{Re}_{a}=858, k_{\mathrm{a}}=3.49$. Sensitivity: a to a 3-D steady wall-normal blowing and suction of spanwise wavenumber $k_{a}$, as quantified by the adjoint pressure $p_{k, a}^{\dagger}$, plotted as a function of the horizontal position $x$ at the upper wall (right part of the diagram) and of the vertical position $y$ at the lateral wall (left part of the diagram with flipped $y$ coordinates). The vertical grey solid and dashed lines mark, respectively, the position of the first reattachment point and of the nozzle edge at $x= \pm e / 2$ and $y=H$. $\mathbf{b}$ to a bulk forcing of same spanwise wavenumber, as quantified by the adjoint $x$-velocity component $u_{k, a}^{\dagger}$

low-level receptivity is made visible by an appropriate choice of the colour look-up table, where the pale blue hue represents negative values about $10 \%$ of the minimum. Finally, the adjoint global mode vanishes downstream of the second recirculating bubble. This specific localization of the adjoint global mode, which differs from that documented for the direct global mode, is due to the so-called convective non-normality of the linearized Navier-Stokes operator [5,6,17]. It can be easily understood by comparing the direct (4) and adjoint (10) equations: the terms accounting for the advection of disturbances by the base flow, $\boldsymbol{\nabla} \hat{\boldsymbol{u}} \cdot \boldsymbol{U}$ for the direct equations and $-\nabla \hat{\boldsymbol{u}}^{\dagger} \cdot \boldsymbol{U}$ for the adjoint equations, have opposite signs. Physically, this indicates that direct perturbations are convected downstream by the base flow, and that adjoint perturbations are convected upstream, hence explaining their different spatial distributions [5].

\subsection{Wavemaker and control via a force-velocity coupling}

The adjoint analysis is also useful to identify the region of the flow which acts as the 'wavemaker' of the instability, i.e. the region where a given eigenvalue is most sensitive to a small modification of the stability problem [5,10]. Giannetti and Luchini [11] have considered the effect of a small modification of the evolution operator under the form of a localized 'force-velocity' coupling, that can also be viewed as a feedback induced by an actuator located at the same station as the sensor. The forcing is defined by

$$
\delta \hat{\boldsymbol{f}}(x, y)=C \mathcal{Q} \hat{\boldsymbol{u}}(x, y) \delta\left(x-x_{c}, y-y_{c}\right)
$$

where $C$ is a constant representing the magnitude of the feedback, $\mathcal{Q}$ is a unitary $3 \times 3$ matrix, $\delta(x, y)$ is a Dirac distribution and $\left(x_{c}, y_{c}\right)$ indicates the position where the feedback acts. Using a perturbation approach of the linear evolution operator (valid only when $C$ is small) along with a standard Cauchy-Schwartz inequality yields simply

$$
|\delta \lambda|=\left|\frac{C}{A}\right|\left|\hat{\boldsymbol{u}}^{\dagger}\left(x_{c}, y_{c}\right) \cdot \mathcal{Q} \hat{\boldsymbol{u}}\left(x_{c}, y_{c}\right)\right| \leq\left|\frac{C}{A}\right|\left\|\hat{\boldsymbol{u}}^{\dagger}\left(x_{c}, y_{c}\right)\right\|\left\|\hat{\boldsymbol{u}}\left(x_{c}, y_{c}\right)\right\| .
$$

The sensitivity of the eigenvalue to such a local feedback is therefore non-vanishing in the region where the product of the modulus of the direct and adjoint global modes is not zero, as stated by Giannetti and Luchini [11]. This overlapping region may thus be viewed as a first definition of the wavemaker of the instability. The magnitude of the product between the modulus of the direct and adjoint global modes, i.e. $\left\|\hat{\boldsymbol{u}}_{\boldsymbol{k}, \boldsymbol{a}}^{\dagger}(x, y)\right\|\left\|\hat{\boldsymbol{u}}_{k, a}(x, y)\right\|$, is shown in Figs. 7a. As a result of the convective non-normality, the product is almost zero everywhere in the flow, except in the core of the first recirculating bubble, where the largest values are reached. 
(a)

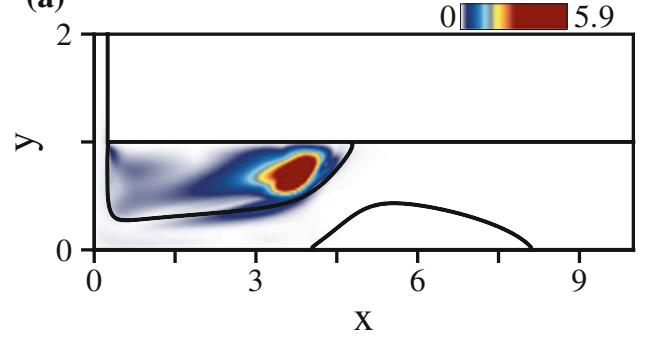

(b)

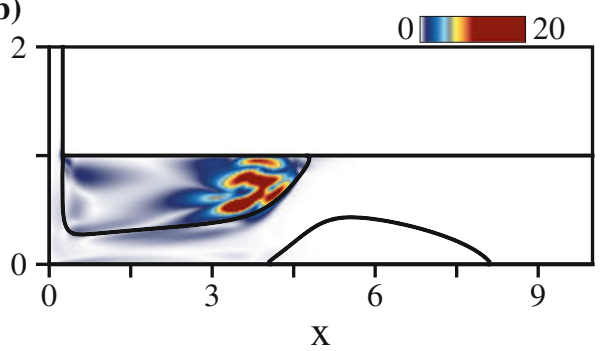

Fig. 7 Antisymmetric mode at the threshold of instability- $\operatorname{Re}_{a}=858, k_{a}=3.49$. Sensitivity: a to local modifications of the linearized evolution operator corresponding to a local 'force-velocity' coupling [11], as quantified by the field $\left\|\hat{\boldsymbol{u}}_{\boldsymbol{k}, \boldsymbol{a}}^{\dagger}(x, y)\right\|\left\|\hat{\boldsymbol{u}}_{k, a}(x, y)\right\|, \mathbf{b}$ to base flow modifications, as quantified by the field $\left\|\hat{\mathcal{S}}_{k, a}(x, y)\right\|$

\section{Adjoint base flow and 2-D stationary control}

By extrapolation on the above 'force-velocity' coupling results, Giannetti and Luchini [11] propose that base flow modifications should occur in the so-defined wavemaker region to affect the flow stability. This interpretation is only qualitative since the modified evolution operator does not involve solely the perturbation velocity, but also its gradients. Such base flow modifications do not result in a local coupling since the pressure perturbation encompasses non-local effects. This concept of sensitivity has been extended to assess how prescribed steady base flow modifications or addition of a steady bulk force may alter the stability properties of a flow, leading to the definition of the so-called sensitivity to base flow modifications or sensitivity to a steady force, respectively $[12,16]$.

\subsection{Wavemaker and sensitivity to base flow modifications}

We investigate first the sensitivity of the leading eigenvalue to an arbitrary base flow modification $\boldsymbol{\delta} \boldsymbol{U}$, as originally formulated by Bottaro et al. [3] and further discussed by Marquet et al. [16]. This analysis is appropriate to identify theoretically which mechanisms are responsible for the instability, as it investigates how and where the growth rate of the unstable modes is affected by changes in the shape of the base flow profiles. Such a property is crucial when departure from the ideal base flow conditions is concerned, for instance owing to imperfections inherent to experimental set-ups. This region can thus be viewed as a second definition of the wavemaker of the instability.

Marquet et al. [16] have shown that the variation $\delta \lambda$ induced by a base flow modification $\delta \boldsymbol{U}$ is such that

$$
\delta \lambda=\int_{\Omega}(\underbrace{-\nabla \hat{\boldsymbol{u}}^{* T} \cdot \hat{\boldsymbol{u}}^{\dagger}}_{\text {Advection }}+\underbrace{\nabla \hat{\boldsymbol{u}}^{\dagger} \cdot \hat{\boldsymbol{u}}^{*}}_{\text {Production }}) \cdot \boldsymbol{\delta} \boldsymbol{U} \mathrm{d} \Omega .
$$

Note that in the general case, the sensitivity field $\hat{\mathcal{S}}=-\nabla \hat{\boldsymbol{u}}^{* T} \cdot \hat{\boldsymbol{u}}^{\dagger}+\nabla \hat{\boldsymbol{u}}^{\dagger} \cdot \hat{\boldsymbol{u}}^{*}$ is complex. However, we deal here with a stationary mode, i.e. the eigenvalue is real and the eigenvector chosen as $\hat{\boldsymbol{q}}_{k, a}=\left(\hat{u}_{k, a}, \hat{v}_{k, a}, \mathbf{i} \hat{w}_{k, a}, \hat{p}_{k, a}\right)^{\mathrm{T}}$ is real. Consequently, relation (13) shows that, no matter the base flow modification, the mode keeps being stationary since $\delta \omega_{k, a}=0$, and (13) can be used to compute directly the growth rate variation $\delta \sigma_{k, a}$. Figure $7 \mathrm{~b}$ presents the spatial distribution of magnitude of the flow field $\left\|\hat{\mathcal{S}}_{k, a}\right\|$. The colour look-up table has been set-up so as to enhance the active zones where the growth rate $\sigma_{k, a}$ is most sensitive. The magnitude of sensitivity is almost zero everywhere in the flow, except in the core of the first recirculating bubble, which thus acts as the wavemaker region. Since the sensitivity field $\hat{\mathcal{S}}$ is given by local products of the adjoint global mode with gradients of the direct global mode (and vice-versa), its spatial distribution therefore differs from that of the only direct and adjoint modes, localized, respectively, downstream and upstream as a result of the convective non-normality. A 2-D steady control of the global mode $\hat{\boldsymbol{q}}_{k, a}$ should therefore induce modifications of the base flow close to the core of the first recirculation to achieve a maximum stabilizing or destabilizing effect. Note that since the direct and adjoint global modes have the same symmetry, the sensitivity field is symmetric, whatever the symmetry of the global mode. This implies that only the symmetric component of a base flow modification affects a given eigenvalue. In particular, it means that the eigenvalue spectrum is insensitive to 
antisymmetric base flow perturbations, as for instance the addition of a small but finite amplitude of the 2-D antisymmetric global mode $\hat{\boldsymbol{q}}_{0, a}$ (Sect. 3.2).

\subsection{Wavemaker and sensitivity to a steady force}

In the perspective of control, the previous sensitivity analysis fails to predict how to produce physically relevant base flow modifications susceptible to modify the stability of a global mode. To this end, we consider now a small-amplitude 2-D stationary forcing, occurring through a small amplitude bulk force $\boldsymbol{\delta} \boldsymbol{F}$ or a blowing and suction velocity at the wall $\delta \boldsymbol{U}_{W}$, both being homogeneous along $z$, as formulated by Hill [12] and Marquet et al. [16]. We assume that the forcing acts at the base flow level, the disturbance equations (4) being unchanged. The base flow equations now read

$$
\nabla \cdot \boldsymbol{U}=0, \quad \nabla \boldsymbol{U} \cdot \boldsymbol{U}+\nabla P-R e^{-1} \nabla^{2} \boldsymbol{U}=\boldsymbol{\delta} \boldsymbol{F}
$$

along with the boundary condition $\boldsymbol{U}=\boldsymbol{\delta} \boldsymbol{U}_{W}$ on $\Gamma_{\mathrm{c}}$. Hill [12] and Marquet et al. [16] have shown that the variation $\delta \lambda$ is such that

$$
\delta \lambda=\int_{\Omega} \boldsymbol{U}^{\dagger} \cdot \boldsymbol{\delta} \boldsymbol{F} \mathrm{d} \Omega+\int_{\Gamma_{\mathrm{c}}}\left(P^{\dagger} \boldsymbol{n}+R e^{-1} \nabla \hat{\boldsymbol{U}}^{\dagger} \cdot \boldsymbol{n}\right) \cdot \boldsymbol{\delta} \boldsymbol{U}_{W} \mathrm{~d} l .
$$

In (15), $\boldsymbol{Q}^{\dagger}=\left(\boldsymbol{U}^{\dagger}, P^{\dagger}\right)^{\mathrm{T}}$ is the adjoint base flow, solution of the 2-D, steady, forced, adjoint problem

$$
\nabla \cdot \boldsymbol{U}^{\dagger}=0, \quad \nabla \boldsymbol{U}^{\mathrm{T}} \cdot \boldsymbol{U}^{\dagger}-\nabla \hat{\boldsymbol{U}}^{\dagger} \cdot \boldsymbol{U}+\nabla P^{\dagger}-R e^{-1} \nabla^{2} \boldsymbol{U}^{\dagger}=\hat{\mathcal{S}}
$$

along with homogeneous boundary conditions. It should be emphasized that problem (16) is forced by the sensitivity field $\hat{\mathcal{S}}$ that depends on the 3-D global mode to be controlled. As mentioned previously, we deal here with stationary modes only. The sensitivity field $\hat{\mathcal{S}}_{k, a}$ being real, the adjoint base flow $\hat{\boldsymbol{Q}}_{k, a}^{\dagger}$ is also real. Relation (15) then shows that the forced eigenmode keeps being stationary, and (15) may be understood as a real equation allowing to compute the growth rate variation $\delta \sigma_{k, a}$.

\subsection{Wall forcing}

In the case of a wall forcing, the variation of the growth rate is due to an inviscid contribution accounted for by the adjoint base flow pressure $P^{\dagger}$, and a viscous contribution weighted by the inverse of the Reynolds number. We have checked that the latter viscous contribution is negligible, meaning that the flow is receptive only to a wall-normal steady flux, and that the forcing is efficient only in regions of the wall where the magnitude of $P^{\dagger}$ is large.

The distributions of the adjoint base flow pressure $\hat{P}_{k, a}^{\dagger}$ at the lateral and upper wall are shown in Fig. $8 \mathrm{a}$, where the vertical grey line in the $x$-half plane indicates the position of the first reattachment point and the dashed lines stand for the position of the nozzle edge. On the lateral wall (left part of Fig. 8a), the adjoint pressure distribution increases close to the edge, where the maximum value is reached. This sensitivity to a 2-D steady mass flux in the inlet channel is in a way trivial, as it acts through an increase in the mean flow rate. However, the values found on the lateral wall remain significantly lower than that found along the upper wall (right part of Fig. 8a), where the magnitude of adjoint pressure remains significant in the whole first recirculating bubble, and decreases as one moves further downstream. Blowing and suction at the wall in the first recirculation bubble acts in a more subtle way by modifying the flow within the region which has been shown to act precisely as the wavemaker of the instability. Again, it is important to note that the sensitivity field is symmetric. If wall forcing through blowing and suction is considered, this means that the same efficiency will be achieved applying a velocity $\delta \boldsymbol{U}_{W}$ on one side of the configuration, or a velocity $\delta \boldsymbol{U}_{W} / 2$ on both sides. 
(a)

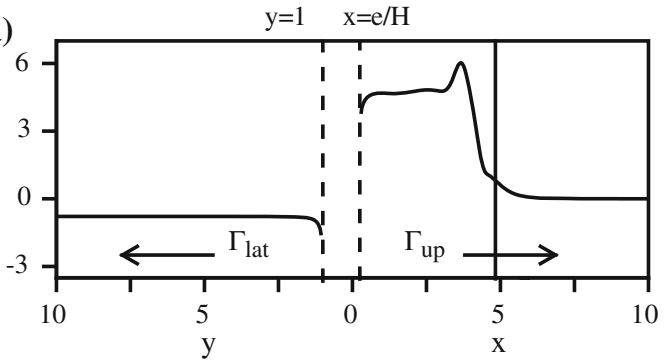

(b)

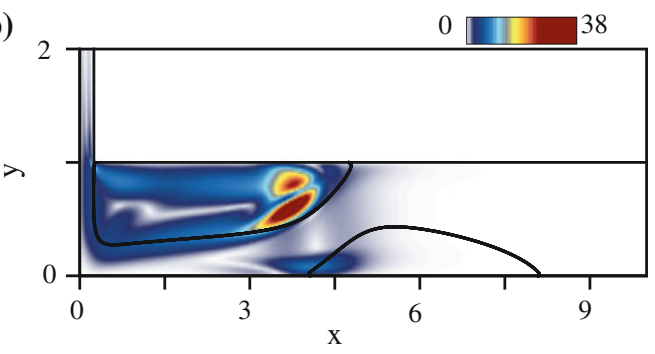

Fig. 8 Antisymmetric mode at the threshold of instability-Re $e_{a}=858, k_{a}=3.49$. Sensitivity: a to a 2-D steady wall-normal blowing and suction, as quantified by the adjoint base flow pressure $\hat{P}_{k, a}^{\dagger}$, plotted as a function of the horizontal position $x$ at the upper wall (right part of the diagram) and of the vertical position $y$ at the lateral wall (left part of the diagram with flipped $y$ coordinates). The vertical grey solid and dashed lines mark, respectively, the position of the first reattachment point and of the nozzle edge at $x= \pm e / 2$ and $y=H$. b to a 2-D steady force, as quantified by the field $\left\|\hat{\boldsymbol{U}}_{k, a}^{\dagger}(x, y)\right\|$

\subsection{Bulk forcing}

In the case of a bulk forcing, the variation of the growth rate depends on the magnitude of the adjoint base flow velocity field $\hat{\boldsymbol{U}}_{k, a}^{\dagger}$. The magnitude of sensitivity, as quantified by the field $\left\|\hat{\boldsymbol{U}}_{k, a}^{\dagger}\right\|$, is weak but non-zero in the vicinity of the separation point at the lower wall and in the region between the two recirculations. Still, the maximum value is reached in the core of the first recirculating bubble, as shown in Fig. 8b. This flow region, which is most sensitive to a 2-D steady forcing, may also be viewed as a third definition of the wavemaker of the instability. It is consistent with the other two definitions considered previously, namely the flow region which is most sensitive to a local 'force-velocity' coupling (Fig. 7a) and to a modification of the base flow (Fig. 7b), all three singularizing out the upper recirculation region.

\section{Application to open-loop control by a small flat-plate airfoil}

We apply now the previous theoretical analysis to investigate the effect of a small control device, chosen as a flat-plate airfoil located at the position $\left(x_{c}, y_{c}\right)$, on the stability of the 3-D global mode $\hat{\boldsymbol{q}}_{k, a}$. The presence of the device is modelled only by the force it exerts on the flow, in the present case a pure lift force, without any change of the domain topology. This contrasts with previous studies where control was carried out by introducing a small cylinder modelled by a pure drag force $[12,16]$. The control by an airfoil has the advantage that it requires no energy to be put into the flow, the work associated to the steady lift force being zero.

The force induced by the airfoil reads

$$
\boldsymbol{\delta} \boldsymbol{F}(x, y)=-\frac{1}{2} C_{\mathrm{L}} l\|\boldsymbol{U}(x, y)\| \boldsymbol{e}_{z} \times \boldsymbol{U}(x, y) \delta\left(x-x_{c}, y-y_{c}\right),
$$

where $C_{\mathrm{L}}$ is a lift coefficient, depending on the angle of attack of the flow relative to the airfoil. In the limit of small angle of attacks, it can be written as

$$
C_{\mathrm{L}}=2 \pi \frac{\boldsymbol{U}\left(x_{c}, y_{c}\right) \cdot \boldsymbol{n}_{L}}{\left\|\boldsymbol{U}\left(x_{c}, y_{c}\right)\right\|}
$$

where $\boldsymbol{n}_{L}$ is the vector normal to the airfoil. In the following, we present results pertaining to the case of a single airfoil of length $l=0.1 \mathrm{H}$ positioned in the horizontal channel, with an incidence $\alpha=-10^{\circ}$ defined relative to the horizontal direction. Should the airfoil be positioned in the inlet section (not shown here), then the incidence would have to be changed from $\alpha$ to $\alpha-\pi / 2$ for the results to be comparable. Since it has been mentioned previously that a given bulk force acts only through its symmetric component, it should be kept in mind that for a given variation $\delta \sigma$ documented in the following, the variation $2 \delta \sigma$ can be obtained by positioning an identical airfoil at the symmetric position $\left(-x_{c}, y_{c}\right)$, but with the incidence $\pi-\alpha$. Again, this requires no supplemental energy to be put into the flow. On the contrary, no effect would be observed by setting both airfoils with the same incidence $\alpha$. 


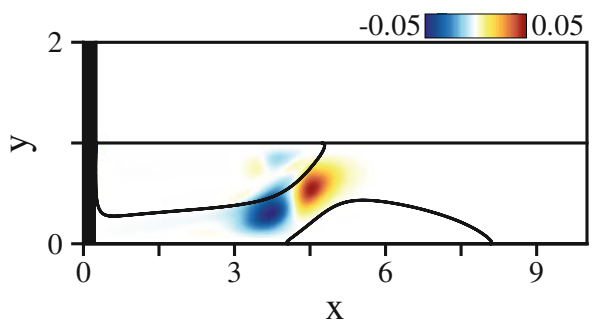

Fig. 9 Antisymmetric mode at the threshold of instability-Re $e_{a}=858, k_{a}=3.49$. Growth rate variation $\delta \sigma\left(x_{c}, y_{c}\right)$ obtained by use of a small control airfoil of length $l=0.1 \mathrm{H}$ placed in the horizontal channel, whose presence is modelled by the lift force (17). The incidence with respect to the horizontal direction is set to $\alpha=-10^{\circ}$. The black shaded area corresponds to the inlet section $\left|x_{c}\right| \leq e$ where the results are not physically relevant

For each position of the airfoil $\left(x_{c}, y_{c}\right)$, the growth rate variation $\delta \sigma_{k, a}$ is given by

$$
\delta \sigma_{k, a}\left(x_{c}, y_{c}\right)=-\frac{1}{2} C_{\mathrm{L}} l\left\|\boldsymbol{U}\left(x_{c}, y_{c}\right)\right\|\left(\boldsymbol{U}\left(x_{c}, y_{c}\right) \times \hat{\boldsymbol{U}}_{k, a}^{\dagger}\left(x_{c}, y_{c}\right)\right) \cdot \boldsymbol{e}_{z} .
$$

A map of the variation $\delta \sigma_{k, a}\left(x_{c}, y_{c}\right)$ is depicted in Fig. 9 for $\left|x_{c}\right| \geq e$. The lift force has a significant stabilizing effect if the airfoil is placed between both recirculating bubbles. Interestingly, this specific flow region is not the one where the magnitude of sensitivity is maximum, as the effect of the force on the growth rate does not only depend on the magnitude of the sensitivity function, which is a vector field, but also on the magnitude of the lift force and on its orientation with respect to the sensitivity function. These results outline the complex effect of lift forcing, as the flow region located just downstream contributes to a destabilization of the global mode of comparable magnitude. Moreover, these two regions exchange roles if the incidence of the airfoil is reversed (in the present case, set to $\alpha=+10^{\circ}$ ).

This variation of the growth rate $\delta \sigma_{k, a}$ due to the steady force applied by the control airfoil may also be computed in two steps, by first computing explicitly the base flow modification $\delta \boldsymbol{U}_{\boldsymbol{F}}$ induced by the 2-D steady forcing. This is done by solving the forced linearized equations

$$
\boldsymbol{\nabla} \cdot \boldsymbol{\delta} \boldsymbol{U}_{\boldsymbol{F}}=0, \quad \boldsymbol{\nabla} \boldsymbol{U} \cdot \boldsymbol{\delta} \boldsymbol{U}_{\boldsymbol{F}}+\nabla \boldsymbol{\delta} \boldsymbol{U}_{\boldsymbol{F}} \cdot \boldsymbol{U}+\nabla \delta P_{F}-R e^{-1} \nabla^{2} \delta \boldsymbol{U}_{\boldsymbol{F}}=\boldsymbol{\delta} \boldsymbol{F},
$$

with homogeneous boundary conditions. To obtain a map of the growth rate variation similar to that shown in Fig. 9, this equation has to be solved for each location of the control airfoil. On the contrary, the sensitivity to a 2-D steady force approach allows to compute all possible locations in a single shot, as the base flow modification is only implicitly taken into account through the forcing sensitivity term $\hat{\mathcal{S}}$. Once the base flow modification is computed from (20) for a specific location of the force $\boldsymbol{\delta} \boldsymbol{F}$, the modification of the growth rate is retrieved using the sensitivity to base flow modifications which is real here:

$$
\delta \sigma_{k, a}=\int_{\Omega} \hat{\mathcal{S}}_{k, a} \cdot \delta \boldsymbol{U}_{\boldsymbol{F}} \mathrm{d} \Omega .
$$

Computing the base flow modifications induced by the control airfoil is useful to understand the underlying physical mechanisms, as Marquet et al. [16] have pointed out that the sensitivity function $\hat{\mathcal{S}}$ is made of two terms stemming from two different physical origins. The first term, $-\nabla \hat{\boldsymbol{u}}^{* T} \cdot \hat{\boldsymbol{u}}^{\dagger}$, originates from the term of advection of perturbations by the base flow $\nabla \boldsymbol{U} \cdot \hat{\boldsymbol{u}}$, while the second term, $\nabla \hat{\boldsymbol{u}}^{\dagger} \cdot \hat{\boldsymbol{u}}$, originates from the term of production of perturbations by the base flow $\nabla \hat{\boldsymbol{u}} \cdot \boldsymbol{U}$. The stabilizing effect of the airfoil can then be investigated in terms of the competition between advection and production of disturbances, by decomposing the growth rate variation $\delta \sigma_{k, a}$ into its advection and production components, i.e. $\delta \sigma_{k, a}=\delta^{\mathrm{T}} \sigma_{k, a}+\delta^{\mathrm{P}} \sigma_{k, a}$ with

$$
\delta^{\mathrm{T}} \sigma_{k, a}=\int_{\Omega}\left(-\nabla \hat{\boldsymbol{u}}_{\boldsymbol{k}, \boldsymbol{a}}^{T} \cdot \hat{\boldsymbol{u}}_{\boldsymbol{k}, \boldsymbol{a}}^{\dagger}\right) \cdot \boldsymbol{\delta} \boldsymbol{U}_{\boldsymbol{F}} \mathrm{d} \Omega, \quad \delta^{\mathrm{P}} \sigma_{k, a}=\int_{\Omega}\left(\nabla \hat{\boldsymbol{u}}_{\boldsymbol{k}, \boldsymbol{a}}^{\dagger} \cdot \hat{\boldsymbol{u}}_{k, a}\right) \cdot \boldsymbol{\delta} \boldsymbol{U}_{\boldsymbol{F}} \mathrm{d} \Omega,
$$

since all fields here are real.

The control airfoil is now placed at the station $\left(x_{c}, y_{c}\right)=(3.6,0.3)$, for which the stabilizing effect achieved is close to the maximum. In practice, the base flow modification $\delta \boldsymbol{U}_{\boldsymbol{F}}$ induced by the airfoil has been 
computed by approximating the lift force (17) by a suitably normalized gaussian function of width 0.0125 centered at $\left(x_{c}, y_{c}\right)$, so as to smooth out the Dirac distribution in the numerics. We obtain consistent results, with $\delta \sigma_{k, a}=-0.046888$ using the sensitivity to a steady force and $\delta \sigma_{k, a}=-0.046887$ using the sensitivity to base flow modifications. This validates the present computations, since both the values are identical down to the fifth digit. Decomposition (22) gives $\delta^{\mathrm{T}} \sigma_{k, a}=-0.178$ and $\delta^{\mathrm{P}} \sigma_{k, a}=+0.131$, meaning that the presence of the airfoil enhances both the advection (stabilizing) and the production (destabilizing) of disturbances, the overall stabilizing effect coming from the strength of the advection effect.

In practice, the effect of the airfoil is two-fold, as it modifies the stability of the global mode considered not only through a 2-D steady force inducing a modification of the base flow, but also through a feedback control on the global mode. Indeed, the presence of the mode at any arbitrarily small amplitude creates a modulation of the lift force with the same amplitude [12]. This results in the existence of a feedback force depending linearly on the disturbance quantities, which can be written as

$$
\boldsymbol{\delta} \hat{\boldsymbol{f}}(x, y)=-\frac{1}{2} l\left[C_{\mathrm{L}}\|\boldsymbol{U}\| \boldsymbol{e}_{z} \times \hat{\boldsymbol{u}}+\left(C_{\mathrm{L}}\|\hat{\boldsymbol{u}}\|+\frac{\partial C_{\mathrm{L}}}{\partial\|\boldsymbol{U}\|} \boldsymbol{U} \cdot \hat{\boldsymbol{u}}\right) \boldsymbol{e}_{z} \times \boldsymbol{U}\right] \delta\left(x-x_{c}, y-y_{c}\right),
$$

where the term $\partial C_{\mathrm{L}} / \partial\|\boldsymbol{U}\|$ accounting for the modification in the lift coefficient can be computed by linearization of (18). From a physical point of view, the first term in (23) corresponds to a modulation in the direction of the lift force, whereas the last two terms correspond to a modulation in the amplitude of the force. Relation (23) is linear in $\hat{\boldsymbol{u}}(x, y)$ and may be written formally as

$$
\boldsymbol{\delta} \hat{\boldsymbol{f}}(x, y)=\mathcal{F}(x, y) \hat{\boldsymbol{u}}(x, y) \delta\left(x-x_{c}, y-y_{c}\right),
$$

where $\mathcal{F}$ is a $3 \times 3$ matrix depending on the position through the base flow quantities. If $l$ is small, this feedback acts as a weak perturbation of the linearized evolution operator and the results pertaining to the 'force-velocity' coupling hold, in particular relation (12). This force thus induces an additional shift in the growth rate

$$
|\delta \sigma|=\left|\frac{1}{A}\right|\left|\hat{\boldsymbol{u}}^{\dagger}\left(x_{c}, y_{c}\right) \cdot \mathcal{F}\left(x_{c}, y_{c}\right) \hat{\boldsymbol{u}}\left(x_{c}, y_{c}\right)\right| \leq \frac{\left\|\mathcal{F}\left(x_{c}, y_{c}\right)\right\|_{\infty}}{|A|}\left\|\hat{\boldsymbol{u}}^{\dagger}\left(x_{c}, y_{c}\right)\right\|\left\|\hat{\boldsymbol{u}}\left(x_{c}, y_{c}\right)\right\|,
$$

where \|\|$_{\infty}$ denotes the infinite norm of the $3 \times 3$ matrix. The feedback induced by the addition of the airfoil will therefore affect the growth rate only in the overlapping region where the field $\left\|\hat{\boldsymbol{u}}_{\boldsymbol{k}, \boldsymbol{a}}^{\dagger}\left(x_{c}, y_{c}\right)\right\|\left\|\hat{\boldsymbol{u}}_{k, a}\left(x_{c}, y_{c}\right)\right\|$ is non-zero, as already discussed in Fig. 7a. The above computations have only taken into account the effect of the base flow modification induced by the steady component of the lift force induced by the airfoil. A comparison of these two effects has been recently carried out for the control of the cylinder wake by means of a small cylinder $[15,17]$, but such a detailed investigation lies out of the scope of this study.

\section{Conclusion}

We have presented a theoretical study of a plane confined impinging jet exhibiting two distinct recirculating bubbles. A linear global stability analysis has been carried out, which shows that the flow is unstable to 3-D antisymmetric and symmetric steady global modes. In the simple case where buoyancy effects are neglected, this 3-D instability induces a significant spanwise modulation of the wall heat flux over a large distance. It also shows the existence of weakly damped 2-D modes, whose effect is to induce antisymmetric and symmetric shifts of the separation and reattachment points, and makes the time simulation of this configuration computationally expensive.

The use of adjoint global modes and sensitivity analyses has allowed to identify different flow regions that are of particular interest in the perspective of control, suggesting different locations of the actuator depending on the control method. In the case of a 'force-velocity' coupling where a local bulk force applies a small feedback on the global mode through a sensor located as the actuator position, the sensor/actuator should be placed in the overlapping region between the direct and adjoint global modes, presently located within the first recirculating bubble. For wall forcing, an actuator inducing a 3-D blowing and suction should be positioned along the lateral walls close to the jet exit, where the level of adjoint pressure is maximum. If 2-D steady control is considered, forcing in the bulk should act so as to modify the base flow in the first recirculating bubble to obtain a large impact on the dynamics. For 2-D steady wall forcing, the actuator should be positioned anywhere in the first recirculating bubble, along the upper wall, where the adjoint base flow pressure is maximum. 
In practice, a 2-D steady bulk forcing may be achieved by introducing a small device in the flow. As discussed in $[12,15,17]$, such a device has two distinct physical effects, as it results in a 2-D steady force that modifies the base flow and a harmonic force that induces a 'force-velocity' feedback on the global mode. We have applied our theoretical framework to the case of a small airfoil acting through a pure lift force. The main interest of this novel approach, compared to the classical approach based on the use of a small cylinder, is that the work associated to the force is zero, meaning that no energy is put into the flow by the control. We show that the airfoil has a stabilizing effect if positioned between the first and second recirculating bubbles, owing to the strengthening of the disturbances advection.

Acknowledgements The authors gratefully acknowledge enlightening and stimulating discussions with D. Sipp and O. Marquet.

\section{References}

1. Barkley, D., Gomes, M., Henderson, R.: Three-dimensional instability in flow over a backward-facing step. J. Fluid Mech. 473, 167-190 (2002)

2. Bayly, B.: Three-dimensional centrifugal-type instabilities in inviscid two-dimensional flows. Phys. Fluids 31, 56-64 (1988)

3. Bottaro, A., Corbett, P., Luchini, P.: The effect of base flow variation on flow stability. J. Fluid Mech. 476, 293-302 (2003)

4. Chiriac, V., Ortega, A.: A numerical study of the unsteady flow and heat transfer in a transitional confined slot jet impinging on an isothermal surface. Int. J. Heat Mass Transf. 45, 1237-1248 (2002)

5. Chomaz, J.M.: Global instabilities in spatially developing flows: non-normality and nonlinearity. Ann. Rev. Fluid Mech. 37, 357-392 (2005)

6. Chomaz, J.M., Huerre, P., Redekopp, L.: The effect of nonlinearity and forcing on global modes. In: Coulet, P., Huerre, P. (eds.) New Trends in Nonlinear Dynamcs and Pattern-Forming Phenomena: The Geometry of Nonequilibrium. NATO ASI Series B, vol. 237, pp. 259-274. Plenum, New York (1990)

7. Davis, T.A.: A column pre-ordering strategy for the unsymmetric-pattern multifrontal method. ACM Trans. Math. Softw. 30(2), 165-195 (2004)

8. Ehrenstein, U., Gallaire, F.: On two-dimensional temporal modes in spatially evolving open flows: the flat-plate boundary layer. J. Fluid Mech. 536, 209-218 (2005)

9. Gallaire, F., Marquillie, M., Ehrenstein, U.: Three-dimensional transverse instabilities in detached boundary layers. J. Fluid Mech. 571, 221-233 (2007)

10. Giannetti, F., Luchini, P.: Receptivity of the circular cylinder's first instability. In: Proceedings of the 5th European Fluid Mechanics Conference, Toulouse (2003)

11. Giannetti, F., Luchini, P.: Structural sensitivity of the first instability of the cylinder wake. J. Fluid Mech. 581, 167-197 (2007)

12. Hill, D.: A theoretical approach for analyzing the restabilization of wakes. Technical Report 103858, NASA (1992)

13. Lee, H., Yoon, H., Ha, M.: A numerical investigation on the fluid flow and heat transfer in the confined impinging slot jet in the low reynolds number region for different channel heights. Int. J. Heat Mass Transf. 51, 4055-4068 (2008)

14. Lifschitz, A., Hameiri, E.: Local stability conditions in fluid dynamics. Phys. Fluids A 3, 2644-2651 (1991)

15. Luchini, P., Giannetti, F., Pralits, J.: Structural sensitivity of linear and nonlinear global modes. AIAA Paper 2008-4227 (2008)

16. Marquet, O., Sipp, D., Jacquin, L.: Sensitivity analysis and passive control of the cylinder flow. J. Fluid Mech. 615, 221-252 (2008)

17. Marquet, O., Sipp, D., Jacquin, L., Chomaz, J.M.: Multiple timescale and sensitivity analysis for the passive control of the cylinder flow. AIAA Paper 2008-4228 (2008)

18. Martin, A.: Heat and mass transfer between impinging gas jets and solid surfaces. Adv. Heat Transf. 13, 1-60 (1977)

19. Schmid, P., Henningson, D.: On the stability of a falling liquid curtain. J. Fluid Mech. 463, 163-171 (2002)

20. Sipp, D., Jacquin, L.: Three-dimensional centrifugal-type instabilities of two-dimensional flows in rotating systems. Phys. Fluids 12, 1740-1748 (2000)

21. Varieras, D.: Étude de l'écoulement et du transfert de chaleur en situation de jet plan confiné. Ph.D. thesis, Université Paul Sabatier-Toulouse III (2000)

22. Varieras, D., Brancher, P., Giovannini, A.: Self-sustained oscillations of a confined impinging jet. Flow Turb. Comb. 78, $1-15$ (2007) 